\title{
MICROENCAPSULATION OF CALCIUM LACTOBIONATE FOR \\ PROTECTION FROM MICROORGANISMS IN A SOLID PHASE FOOD
}

\author{
S. Sáez-Orviz ${ }^{\text {a }}$ P. Camilleria ${ }^{\text {a I. Marcet }}{ }^{\mathrm{a}}$, M. Rendueles ${ }^{\mathrm{a}}$, M. Díaz ${ }^{\mathrm{a} *}$ \\ ${ }^{a}$ Department of Chemical and Environmental Engineering, University of Oviedo, \\ C/Julián Clavería s/n, 33071 Oviedo, Spain. \\ *Corresponding author. \\ E-mail address: mariodiaz@uniovi.es (M. Díaz)
}

\begin{abstract}
Prebiotic compounds may be consumed during storage by the foodstuff's own microflora, so that new processes to protect these bioactive compounds before they are released in the gastrointestinal tract need to be developed. With this aim, lyophilized calcium lactobionate microparticles were designed, employing different coat materials (caseinate, gelatine, gum arabic, maltodextrin and mixtures of gum arabic and maltodextrin), some of which had previously been treated with transglutaminase enzyme. The microparticles were introduced into cottage cheese, as an example of a matrix with low water activity, and were found to exert a successful protective effect against consumption of lactobionate by lactic acid bacteria present in the cheese. Diffusion of the calcium lactobionate to the surface of the microparticles and diffusion of the lactic bacteria into the microparticles were negligible during the tested period. A model was developed to simulate the lactobionate consumption in the microparticles-cheese system. All the microparticles tested had good protective characteristics and no important improvement was observed when transglutaminase was added. Microparticles of sodium caseinate were chosen for the final digestibility analyses. Subsequently, in the digestibility tests, the calcium
\end{abstract}


lactobionate was released from sodium caseinate microparticles in the cottage cheese under both gastric and intestinal conditions. Therefore, this study presents a protective microparticle process for use in functional food products.

Keywords: prebiotic, calcium lactobionate, microencapsulation, cottage cheese, functional dairy product.

\section{Introduction}

Nowadays, there is a great demand for high quality food products with no risk of any ill effects and which, besides satisfying the consumers' nutritional demands, have the capacity to improve their health. To fulfil this perceived need, what has been called "functional food" has received growing attention recently. According the International Life Sciences Institute (ILSI), functional food can be defined as "that which beneficially affects one or more target functions in the body, beyond adequate nutritional effects, in a way that is relevant to either an improved state of health and well-being and/or reduction of risk of disease" [1].

The functional food market is currently growing, and Japan is one of the main producers and consumers. About $45 \%$ of Japanese functional foodstuffs aim to help with the maintenance of good gastrointestinal tract (GIT) conditions [2]. The GIT microbiota is arranged in a complex ecosystem which directly affects human nutrition and health. Thus, it is of interest to increase the number of beneficial bacteria to the point where they represent more than half the population. There are two ways to achieve this goal: using living bacteria, known as probiotics, and/or employing prebiotic compounds [1].

Prebiotics constitute a vast variety of compounds. Within this group, lactose derivates such as lactobionic acid are of great interest. This compound has received attention from 
the food industry due to its properties as an antioxidant, stabilizer and gelling agent [3], but also for its prebiotic effect. CaLb consumption in the colon by the human microflora is capable of improving the growth of some probiotics of the Bifidobacterium genus [4] [5]. Lactobionic acid has been approved for use as calcium lactobionate $(\mathrm{CaLb})$ by the FDA (FDA, 2017), although it is still being studied by European Committees [3].

Functional foods incorporate bioactive compounds which are usually sensitive to chemical and physical factors [7]. Specifically, for prebiotics, introducing them directly into food products leads to the risk of their being consumed by the foodstuff's own microflora. If that happens, the prebiotics will not be able to reach the consumers' GIT and so promote the growth of beneficial probiotics. To deal with the problems of the degradation of bioactive-compounds, the food industry has developed several tools, such as microencapsulation ([7], [8]). There are many microencapsulation techniques, but that of lyophilization allows the creation of microparticles that are stable over time [9]. There have been several studies on the microencapsulation of bioactive compounds, the vast majority of which do not include experimental investigation of the incorporation of microparticles into food matrices. The most usual food matrices employed to test microparticles are dairy products ([10], [11], [7]). The use of low water activity $\left(\mathrm{a}_{\mathrm{w}}\right)$ food matrices such as bread is also common [12], as is the use of cereals.

As far as is known, there are few prebiotic microencapsulated products on the market. In this respect, the purpose of the present research was to develop and characterize CaLb microparticles, employing different coat materials and transglutaminase enzyme (TGase), with the objective of improving the microparticle properties. After analysing the microparticles, they were tested in cottage cheeses as an example of a food matrix with low $\mathrm{a}_{\mathrm{w}}$. The aim was to determine the protective effect of microparticles against the consumption of lactobionic acid by the cottage cheese's own microflora and to generate 
an innovative functional dairy product that could be attractive for both consumers and the food industry.

\section{Materials and methods}

\subsection{Materials}

Several coat materials with different concentrations (w/w) were employed as described by other authors ([9], [13], [14], [15] ). Sodium caseinate (NaCas), maltodextrin (MD) and gelatine (Gel) were purchased at Sigma-Aldrich Chemical Co. (Steinheim, Germany). Gum arabic (GA) was provided by Panreac (Panreac S.A., Barcelona Spain). $\mathrm{CaLb}(\geq 98 \%$ purity) was supplied by Sigma-Aldrich.

\subsection{Microparticle preparation}

Mixtures of $0.05 \mathrm{~g} \mathrm{~mL}^{-1} \mathrm{CaLb}$ and the different coat agent solutions were prepared at a ratio of $1: 3$ and they were frozen at $-80{ }^{\circ} \mathrm{C}$ for 12 hours. Microparticles were produced using the lyophilisation method (Tesltar Cryodos, $0.1 \mathrm{mBar},-70^{\circ} \mathrm{C}$ for 24 hours). After lyophilisation, microparticles were ground and sieved with a pore-size of $355 \mu \mathrm{m}$ and stored in a dry atmosphere until use. The final concentration of $\mathrm{CaLb}$ for each microparticle type after preparation is indicated in Table 1.

Mixtures of NaCas and Gel with the active material were also treated with transglutaminase (TGase), in an attempt to improve their properties. In this case, $\mathrm{pH}$ was adjusted to 7.0 with $1 \mathrm{M} \mathrm{NaOH}$ (Sigma-Aldrich) and TGase was added (0.33 $\mathrm{g} \mathrm{L}^{-1}, 50 \mathrm{U}$ $\mathrm{g}^{-1}$ protein) (Probind TX, BDF ingredients, Spain). The reaction was conducted in an oven at $45^{\circ} \mathrm{C}$ for $90 \mathrm{~min}$. After that time, the temperature was increased to $70{ }^{\circ} \mathrm{C}$ for $10 \mathrm{~min}$ to deactivate the enzyme. As in the case of the other microparticles, they were produced by the lyophilization method. 


\subsection{Microparticle characterization}

\subsubsection{Encapsulation efficiency}

Encapsulation efficiency (EE) was calculated according to Cilek et al. (2012). EE is defined as the ratio of encapsulated active compound content (EAC) to total active compound (TAC). EAC is determined by calculating the difference between TAC and the surface-active compound content (SAC) (Equation 1).

$\mathrm{EE}=[(\mathrm{TAC}-\mathrm{SAC}) / \mathrm{TAC}] \times 100$

To determine SAC, $1 \mathrm{~mL}$ of distilled water was added to $0.005 \mathrm{~g}$ of microparticles and they were gently shaken for 10 minutes. Samples were centrifuged at 13,200 rpm (Centrifuge 5415D, Sigma-Aldrich) for 5 minutes. The supernatant was used to measure SAC as described in section 2.5.

\subsubsection{Solubility}

This test investigates which kind of microparticle prevents further release of CaLb. $1 \mathrm{~mL}$ of distilled water was added to $0.005 \mathrm{~g}$ of each type of microparticle. Samples were incubated at $20{ }^{\circ} \mathrm{C}$ and $300 \mathrm{rpm}$ for $30 \mathrm{~min}$ (Thermomixer, Eppendorf, Hamburg, Germany). After centrifugation (13,200 rpm for $5 \mathrm{~min})$, the supernatant was collected and analysed as described in section 2.5.

\subsubsection{Microstructure characterization}

The morphology of the microparticles was observed using scanning electron microscopy (SEM) (JSM-6610LV, JEOL, USA). Microparticles were mounted on stubs and coated with gold. The surface morphology of the microparticles was observed with magnifications of 100x. 


\subsection{Functionalization of cheese with calcium lactobionate}

\subsubsection{Functional cottage cheese manufacture}

Milk was obtained from a herd of Murciano-Granadino goats from a local farm in San Martín del Rey Aurelio (Asturias, Spain). Cottage cheeses were made with low temperature-long time (LTLT) pasteurised milk, heated at $60{ }^{\circ} \mathrm{C}$ for 25 minutes. This gentle pasteurization process allows some lactic acid bacteria (LAB) to survive, so it was not necessary to use a starter culture. Subsequently, milk was cooled to $34{ }^{\circ} \mathrm{C}$ and rennet $\left(\right.$ Chy-Max ${ }^{\circledR}$, CHR-Hansen, Denmark) was added $\left(0.0225 \mathrm{~g} \mathrm{~L}^{-1}\right)$. After 40 minutes, the curd was cut several times to stimulate syneresis. Approximately $0.005 \mathrm{~g}$ of each microparticle type was mixed carefully with approximately $40 \mathrm{~g}$ of curd. The concentration of $\mathrm{CaLb}$ in the global cheese-microparticles mix is shown in Table 1 for all the tests. Cheeses were stored at $21{ }^{\circ} \mathrm{C}$ for a day and then at $4{ }^{\circ} \mathrm{C}$ during the maturation period for further analysis.

Analysis of microparticles inside functional cottage cheeses was carried out using $5 \mathrm{~g}$ samples of each cheese. $20 \mathrm{~mL}$ of $\mathrm{H}_{2} \mathrm{SO}_{4}(0.013 \mathrm{~N})$ (Sigma-Aldrich) [16] was added and samples were homogenised using a Stomacher ${ }^{\circledR} 80$ (Seward, United Kingdom) for 90 seconds at maximum speed. Afterwards, samples were heated at $64{ }^{\circ} \mathrm{C}$ for 30 minutes. Finally, samples were centrifuged for 10 minutes at 13,200 rpm and the supernatant was stored until analysis as described in section 2.5.

\subsubsection{Textural analysis}

For textural characterisation, a TA.XTplus Texture Analyzer (Stable Systems, Godalming, Surrey, United Kingdom) was employed. Cottage cheese samples were subjected to a penetration test, at room temperature, using the spherical probe SMS P/0.5S with a test speed of $2.0 \mathrm{~mm} \mathrm{~s}^{-1}$ and a load cell of $5 \mathrm{~kg}$. Results are expressed in terms of 
firmness and stickiness values (grams). Experiments were triplicated and reported results correspond to the mean value.

\subsubsection{Digestibility test}

Gastric and intestinal conditions were simulated according to Minekus et al. (2014), with some modifications. The composition of the simulated gastric fluid (SGF) employed was $0.517 \mathrm{~g} \mathrm{~L}^{-1} \mathrm{KCl}, 0.123 \mathrm{~g} \mathrm{~L}^{-1} \mathrm{KH}_{2} \mathrm{PO}_{4}, 2.106 \mathrm{~g} \mathrm{~L}^{-1} \mathrm{NaHCO}_{3}$ and $2.75 \mathrm{~g} \mathrm{~L}^{-1} \mathrm{NaCl}$. Simulated intestinal fluid (SIF) was made by mixing $0.509 \mathrm{~g} \mathrm{~L}^{-1} \mathrm{KCl}, 0.110 \mathrm{~g} \mathrm{~L}^{-1} \mathrm{KH}_{2} \mathrm{PO}_{4}, 11.68 \mathrm{~g}$ $\mathrm{L}^{-1} \mathrm{NaHCO}_{3}$ and $2.24 \mathrm{~g} \mathrm{~L}^{-1} \mathrm{NaCl}$.

For the gastric simulation, 5 grams of cottage cheese were mixed with $7.5 \mathrm{ml} \mathrm{SGF,} 1.6$ mL of pepsin (863 $\mathrm{U} \mathrm{mg}^{-1}$ protein, CAS 9001-75-6, Sigma-Aldrich), with a concentration of $15.15 \mathrm{~g} \mathrm{~L}^{-1}$, using SGF as solvent, $5 \mu \mathrm{L}$ of $\mathrm{CaCl}_{2}$ 0.3M (Sigma-Aldrich) and $0.696 \mu \mathrm{L}$ of distilled water. The $\mathrm{pH}$ was adjusted to 3.0 with $1 \mathrm{M} \mathrm{HCl}$ (Sigma-Aldrich). The mixtures were shaken at $300 \mathrm{rpm}$ and $37^{\circ} \mathrm{C}$ for 90 minutes and samples were collected at 45 and 90 minutes.

After gastric simulation, intestinal simulation was carried out. $4.95 \mathrm{~mL}$ of SIF, bovine chymotrypsin $(0.3 \% \mathrm{w} / \mathrm{v})\left(60 \mathrm{U} \mathrm{mg}^{-1}\right.$ protein, EC 232-671-2, Sigma-Aldrich), porcine pancreatin $(0.1 \%$ w/v) (Sigma-Aldrich), $40 \mu \mathrm{L}$ of $0.3 \mathrm{M} \mathrm{CaCl} 2$ and $1.3 \mathrm{~mL}$ of distilled water were added to the previous mixes. The $\mathrm{pH}$ was adjusted to 7.0 using $1 \mathrm{M} \mathrm{NaOH}$ (Sigma-Aldrich). The mixtures were shaken at $300 \mathrm{rpm}$ and $37^{\circ} \mathrm{C}$ for 2 hours and samples were collected at 15, 30, 45, 60 and 120 minutes. All samples taken at different times were centrifuged $(13,200 \mathrm{rpm}$ for 5 minutes) and filtered with a $0.45 \mu \mathrm{L}$ syringe filter (Whatman, Sigma-Aldrich) before analysis.

\subsubsection{Morphological analysis}


Structural analysis was carried out to search for any changes in the cottage cheese structure when microparticles were added. Cottage cheeses were frozen at $-80^{\circ} \mathrm{C} \mathrm{o} / \mathrm{n}$ and then were lyophilised $\left(0.1 \mathrm{mBar},-70^{\circ} \mathrm{C}\right.$ for 24 hours). Morphology was studied using SEM as described in section 2.3.3.

\subsection{Analytical methods}

CaLb was measured as lactobionic acid using High Performance Liquid Chromatography (HPLC). The system of liquid chromatography employed (Agilent 1200, Agilent Technologies Inc., Santa Clara, CA, USA) was equipped with a Coregel ION 300 column (Teknokroma, Barcelona, Spain) coupled to a refractive index detector. The mobile phase was a sulphuric acid solution $\left(0.450 \mathrm{mmol} \mathrm{L}^{-1}, \mathrm{pH} 3.1\right)$ with a flow rate of $0.3 \mathrm{~mL} \mathrm{~min}^{-1}$ and a column temperature of $75^{\circ} \mathrm{C}$. Data acquisition and analysis were performed with ChemStation software (Agilent).

\section{Results and discussion}

\subsection{Characterization of microparticles}

\subsubsection{Encapsulation efficiency (EE)}

EE indicates the amount of active compound encapsulated with respect to the initial quantity used. Experiments were performed in triplicate and results are shown as the mean value with the standard deviation (Table 2).

The NaCas and Gel microparticles treated with TGase showed an improvement in the EE, NaCas being the better core material (Table 2). TGase catalyses the acetyltransferase reaction (intra or inter chain) between the $\gamma$-carboxamide groups of glutamic residues and the $\varepsilon$-amine groups of the lysine residues [18], which creates crosslinking. It has been shown that treatment with this enzyme improves the microparticle characteristics of 
different kinds of core material, leading to a higher EE [19]. It is also capable of enhancing water retention [20] and as CaLb is a hydrophilic compound, core material treated with TGase achieved a better EE.

In the case of $\mathrm{MD}$ and $\mathrm{GA}$, the $\mathrm{EE}$ values increased when more core material was employed. GA $8 \%$ showed an EE of $88.4 \pm 1.8 \%$, while at $4 \%$ the EE decreased to $64.8 \pm 2.8 \%$. Something similar happened with MD microparticles, where MD $12 \%$ showed a reduction of $10 \%$ in its EE value with respect to MD 16\%. So, the greater the amount of core material, the higher the encapsulation efficiency. Other authors explain it as a faster precipitation of the core material [21].

Mixtures of MD and GA had the highest amount encapsulated and it can be seen that the EE rose when there was a higher proportion of GA. This can be explained by the properties of GA as a stabilizing and emulsifying agent [13] and by the fact that GA has a higher molecular weight, a characteristic that enables it to retain more active material [22].

\subsubsection{Microparticle solubility}

Solubility experiments were performed in triplicate and results are shown as the mean value with the standard deviation (Table 2). Excepting GA:MD mixtures, the rest of the microparticles have a CaLb release of $60 \%$. It is a high percentage, as expected, because most coating materials employed were hydrosoluble.

GA:MD mixtures were extremely water-resistant, but GA and MD separately were not. The two polysaccharides together form water-resistant microparticles, possibly as a result of some interaction that leads to longer polysaccharide chain length, which implies lower solubility [8]. Gel was also soluble even after being treated with TGase and was even less water resistant. TGase activity may form some new bonds but others might be destroyed 
due to physical and chemical factors like $\mathrm{pH}$ or temperature [18] changing the gelatine structure and thus affecting parameters like solubility. NaCas and NaCas-TGase had a mean solubility value of $69.1 \%$. Caseins had a low solubility in an acid environment [23] but the salt form increases solubility.

Excluding GA:MD mixtures, the results demonstrated that these microparticles were not appropriate for addition to food matrixes with high $\mathrm{a}_{\mathrm{w}}$, such as milk, but that they could be effective in food products with a low $\mathrm{a}_{\mathrm{w}}$, like cheeses or bread. This is why microparticles have been used more frequently in such foods. There are several examples of the use of microparticles in such foods; for instance, casein microparticles were used with citric acid for chewing gums [24] or whey and MD microparticles with fruit extract for bread [12].

\subsubsection{Microcapsule morphology}

SEM was employed to study surface morphology (Figure 1). The visual appearance of all the microparticles was similar: a white powder with a particle size $\leq 355 \mu \mathrm{m}$.

There was no morphological difference between the microparticles treated with TGase and those that had not been treated. Only the Gel microparticles had a different appearance, with a less uniform surface and less compact structure (Figure 1C and D), while the others had a generally uniform appearance, despite having irregular shapes, and were smooth and with no pores (Figure 1E-K). Microparticle morphology is related to the active compound and the method employed to produce the microparticles. In this study, after lyophilisation, the microparticles were crushed and sifted and, as a result, all microparticles had a uniform and irregular shape despite the core material. Similar surface morphologies were obtained when biopolymers, such as chitosan, xanthan or $\beta$ - 
cyclodextrin, were employed as coat material with phenolics as active compounds in lyophilised microparticles developed by other authors [25] [26].

\subsection{Functionalization of cheese with microparticles}

Milk and dairy products have been the preferred food matrices for the incorporation of microparticles [7]. Some active compounds added to dairy products are plant-derived [11], vitamins [27] and organic acids [10]. There are very few studies investigating prebiotic microencapsulation. Employing dairy products in which probiotics are present together with a prebiotic that is protected against consumption by the food probiotic allows the production of synbiotic products [28] with interesting health properties and that are appealing to consumers.

\subsubsection{Evolution of $\mathrm{CaLb}$ regarding time}

After being characterized, the microparticles were added to the cheese matrix. CaLb was measured at initial time (t 0 ) and after 5 and 12 days. Experiments were performed in triplicate and results of the evolution with time of the concentration of $\mathrm{CaLb}$ for $\mathrm{NaCas}$, NaCasTG, Gel, GA 4\%, MD $16 \%$ and the positive control (free CaLb without encapsulation in microparticles) are presented in the Figure 2 as the points at 0,5 and 12 days.

Due to the LTLT process, the concentration of CaLb decreased over time because some LAB were still alive inside the cheese matrix. CaLb is used as substrate by some LAB [4] but microparticles provided protective capacity against bacterial consumption, as can be seen. The high consumption of CaLb by the LAB can be observed in the positive control. In the case of the Gelatine $(\mathrm{Gel})$, the protective effect was low and therefore the consumption was relatively high. These results agree with the low EE of the Gel shown in Table 2. The concentrations of CaLb contained in NaCas, GA and MD microparticles 
underwent only very low variations after 12 days, indicating a very low consumption by the $\mathrm{LAB}$, and therefore a good level of protection. A comparison of the results for $\mathrm{NaCas}$ and $\mathrm{NaCasTG}$ indicate a small increase in the degree of protection against consumption when the core material was treated with TGase. For the mixtures GA:MD tested (data not shown) the behaviour was similar to GA and MD alone, no significant variations were found.

The real amount of active compound, $\mathrm{CaLb}$, inside the microparticles was between 0.5 and $2.8 \mathrm{mg} \mathrm{CaLb} \mathrm{g}{ }^{-1}$ of cottage cheese. This may seem a very large variation in $\mathrm{CaLb}$ content, but what is really significant is that even the lowest value is much greater than the amount found in the only CaLb-containing food product marketed today. This is Caucasian yogurt ("Caspian Sea yogurt"), which is sold in Japan, where FOSHU ("Food for specified health use") products have been extensively developed. This is the only yogurt that contains $\mathrm{CaLb}$ and its concentration is approximately $0.45 \mathrm{mg}$ of $\mathrm{CaLb} \mathrm{g}^{-1}$ of yogurt [29]. Protecting CaLb against LAB consumption by employing microparticles allowed an increase around $60 \%$ in the concentration of $\mathrm{CaLb}$ respect the commercial product. The FDA and committees are investigating suitable concentrations for consumption (FDA, 2017) but there is no specific regulation at the moment. There are several studies and some authors have concluded that amounts of up to $24 \mathrm{~g}$ of CaLb per day were well tolerated [4]. So, developing innovative new products with high concentrations of $\mathrm{CaLb}$ could be particularly interesting from a technological point of view. Specifically, NaCas and NaCas-TGase microparticles had a concentration of $1.14 \pm 0.03$ and $1.19 \pm 0.04 \mathrm{mg}$ of $\mathrm{CaLb} \mathrm{g}^{-1}$ of cottage cheese respectively. Casein is found naturally in dairy products as the major protein and therefore, employing NaCas microparticles for developing functional dairy products will be accepted more readily by consumers, as it does not involve the use of foreign proteins or sugars. 


\subsubsection{Modelling of CaLb transport and consumption in cheese}

A schematic description of the experimental work considers two phases, the nanoparticles phase and the cheese phase. The CaLb is present initially in the nanoparticles phase; there will be diffusion, $\mathrm{J}_{1}$, to the surface, where there could be some biodegradation, and then diffusion to the bulk cheese phase, $\mathrm{J} 2$. The biodegradation reactions will take place in the neighbourhood of the interphase and in the cheese bulk. The concentrations of CaLb in the nanoparticles, interphase and cheese bulk will be $c_{p}, c_{s}$, and $c_{b}$, while the concentration of the bacterial population will be assumed as uniform in the cheese phase (Figure 3).

A general mass balance for the cheese phase will consider the accumulation of $\mathrm{CaLb}$ to be equal to its mass transfer $\left(J_{1}=k_{s}\left(\begin{array}{ll}C_{p} & C_{s}\end{array}\right)\right)$ to the surface, minus its consumption at the surface $\left(A k_{s} C_{s} X_{s} Y_{x / s}^{s}\right)$ and in the cheese bulk $\left(V k_{x} X_{s} Y_{x / s}^{s}\right)$, which would give $V_{b} \frac{d c_{b}}{d t}=J_{1} \quad r=J_{1} \quad\left(A k_{s} c_{s} X_{s} Y_{x / s}^{s}\right)+\left(V_{b} k_{x} X_{b} Y_{x / s}^{b}\right)$

$A$ being the interphase surface, $V_{b}$ the volume of the cheese bulk, $K_{s}$ and $K_{x}$ the kinetic constants, $X s$ the biomass in the interphase and $Y_{x / s}$ the biomass/substrate stoichiometric coefficient.

A global mass balance for the cheese-microparticles mix could also be of interest in order to obtain experimental data (Figure 2) for the global concentration $c$. Experiments carried out to investigate the biodegradation of CaLb homogeneously distributed in the cheese have indicated a rapid degradation process, so the reaction would seem to occur near the interphase. Assuming a fast reaction which could take place in the cheese and in the nanoparticles film, and clustering the parameters, simplified equations can be obtained for the analysis of the experimental results. 
A local mass balance for the nanoparticles phase will give $\frac{d C_{p}}{d t}=k^{\prime}\left(c_{p}-c_{s}\right)$

and if $c_{s} 0$ we will obtain $\frac{d C_{p}}{d t}=k^{\prime} c_{p}$

A global mass balance over time gives rise to

$$
V_{p} c_{p o}=V c+\int_{0}^{t} V_{p} \frac{d c_{p}}{d t} d t=V c+\int_{0}^{t} V_{p} k^{\prime} c_{p} d t
$$

The term $c_{p o}$ being the initial concentration of CaLb in the microparticles and $V_{p}$ the volume of the microparticles.

Consequently, $\left(\frac{V_{p}}{V}\right) c_{p o}=c+\int_{o}^{t} k^{\prime} c d t$

This equation can be used to obtain the values of $\mathrm{k}^{\prime}$ from the experimental data of $c$ vs. $t$. When NaCas and NaCasTG microparticles were introduced into the cheese, the values obtained for k' were $4.43 \times 10^{-5} \mathrm{~s}^{-1}$ and $4.40 \times 10^{-5} \mathrm{~s}^{-1}$ respectively, indicating that the use of transglutaminase did not exert an important effect on the protection process. When gum arabic (GA) was used, k' was found to be $4.61 \times 10^{-5} \mathrm{~s}^{-1}$, while the value of $\mathrm{k}^{\prime}$ was $3.03 \times 10^{-5} \mathrm{~s}^{-1}$ with maltodextrin (MD), and if gelatine (Gel) was used for making the microparticles, the value of k' was $3.1 \times 10^{-5} \mathrm{~s}^{-1}$. The values of the fitting of the model to the experimental results are shown in Figure 2, with the simulated lines.

As a comparison, the consumption in the positive control, using the same equation (5) gave $\mathrm{k}^{\prime}$ as $4.66 \times 10^{-5} \mathrm{~s}^{-1}$ when the lactobionate was not encapsulated in the microparticles. Taking into account the concentration of lactobionate in the cheese after 12 days, it can be seen that the microparticles exerted a protective effect, impeding the consumption of this compound by the lactic acid bacteria present in the cheese. 


\subsubsection{Textural characterization of functional cheeses}

To produce attractive food products for consumers, it is particularly important to understand their texture and structure [30] [31]. Textural characterization allows the measurement of the firmness and stickiness of food products. Experiments were performed in triplicate and results are shown as the mean value with the standard deviation (Figure $4 \mathrm{~A}$ and $\mathrm{B}$ ).

In terms of firmness, the negative control (cheese without microparticles) had a mean value of $249.18 \pm 26.55 \mathrm{~g}$ (Figure 4A). All the cheeses had a similar value, except the positive control, Gel and Gel-TGase and GA cheeses. In the case of the positive control, the firmness value was $48 \%$ higher than the negative control due to the increase in free calcium in the matrix. $\mathrm{CaCl}_{2}$ is one of the salts which is added to improve the curdling process. CaLb was added free to positive control cheeses, so calcium was able to enhance the precipitation of casein and the strength of its bonds, leading to a greater value of firmness [32]. Firmness of cheeses with GA microparticles showed an increase of 59\% with respect to the negative control. GA has useful properties in the food sector due to its stabilising capacity [13]. It may stabilize casein-casein bonds, thus increasing the firmness of the matrix. The opposite happens with Gel microparticles where cheese firmness was $32 \%$ lower. The $\mathrm{pH}$ of cheese was between 3.5 and 4.5 . At this $\mathrm{pH}$, the number of basic amino acid residues available to participate in the TGase bond with caseins decreased rapidly and therefore firmness also decreased [33].

Referring to stickiness, the values obtained differed notably from the negative control $(3.498 \pm 0.474 \mathrm{~g})$ (Figure 4B). The highest values were obtained with MD microparticles 
and the mixtures of MD and GA. As there was no difference between the GA microparticle cheeses and the control, it can be assumed that MD was responsible for the increase in stickiness values. The presence of MD in the cheeses raised their moisture content [34] and that could affect the structure of the protein matrix, increasing stickiness. An increase in the stickiness value was also observed with TGase microparticles, particularly with Gel-TGase. In the case of casein microparticles, TGase catalyses bonds between different residues in caseins [18]. The increase in this kind of interaction could have as a result a higher stickiness in the final product.

As there was no great difference between the protective effect exerted by the different microparticles, and in all of them the amount of $\mathrm{CaLb}$ encapsulated was acceptable, as were their textural properties, $\mathrm{NaCas}$ and NaCas-TGase microparticles were the ones chosen for digestibility and cheese morphology analysis.

\subsubsection{Functionalized cheese morphology}

The visual appearance of the functional cheeses was similar to the cottage cheese without microparticles in its structure. Functionalised cottage cheeses were examined for matrix differences with SEM (Figure 5).

There was no difference between the negative control matrix and cheeses functionalised with $\mathrm{NaCas}$ and NaCas-TGase (Figure 5A, C and D, respectively). The morphology results corresponded with textural analysis, since there was no difference in firmness and stickiness values between the negative control and cheese functionalized with $\mathrm{NaCas}$ and NaCas-TGase microparticles.

The matrix of the positive control showed evident changes (Figure 5B). It was more homogeneous, rounded and less rough. This could be due to the presence of free calcium. Calcium promotes the curdling process, helping and improving casein precipitation [32]. 
Free calcium could interact with casein, and so change the matrix structure and textural properties, resulting in a firmer and more compact cheese.

\subsection{Digestibility of microparticles and cheeses}

The digestibility test showed the degree of degradation of microparticles with the aim of discovering whether $\mathrm{CaLb}$ can be released in the GIT to enhance the growth of its microflora. The digestibility test was carried out employing NaCas and NaCas-TGase after characterization of these microparticles and their functionalized cheeses.

NaCas and NaCas-TGase powder was first tested to ensure microparticle degradation. Experiments were performed in triplicate. NaCas-TGase microparticles were more resistant to the acid stomach environment, with a CaLb release of $84.8 \pm 1.2 \%(\mathrm{w} / \mathrm{w})$, while NaCas had a liberation of $99.2 \pm 0.8 \%(w / w)$. Casein microparticles can resist low $\mathrm{pH}$ but they are rapidly digested by pepsin [23]. But nevertheless, when casein is treated with TGase, the microparticles are more resistant to $\mathrm{pH}$ and enzymes due to the cross-linking between proteins [35]. In the intestinal simulation, $\mathrm{CaLb}$ release with $\mathrm{NaCas}$ microparticles was similar to that seen in the stomach, while NaCas-TGase showed a higher degree of liberation $(85.7 \pm 0.3 \%)$. A modification in the $\mathrm{pH}$ from acid (3.0) to neutral (7.0) might encourage a change in the protein structure by breaking bonds formed by TGase and increasing CaLb liberation.

Once it had been proved that the microparticles were degraded, functional cheeses were tested in triplicate. As occurred with microparticle powder, NaCas showed more release than NaCas-TGase $(75.6 \pm 0.7 \%(\mathrm{w} / \mathrm{w})$ versus $63.0 \pm 0.7 \%(\mathrm{w} / \mathrm{w}))$ in the stomach. In the intestine, the liberation was similar, although NaCas-TGase functionalised cheeses had a greater degree of release $(78.8 \pm 2.0 \%(\mathrm{w} / \mathrm{w}))$. CaLb release in the cheese digestibility 
experiments was lower than that observed in the microparticles in suspension. This could be due to the complexity of the food matrix.

\section{Conclusions}

Microparticles made with the different coat materials possessed good EE and morphological properties, although their high solubility in water limits their use to food products with a low $\mathrm{a}_{\mathrm{w}}$. All the microparticles exerted a successful protective effect against LAB consumption when they were added to cottage cheese. NaCas and NaCasTGase were selected for further analysis as the coat material is formed by casein, which is the major protein present in milk. No great differences were observed when TGase was employed. The functional dairy product developed revealed good texture and digestibility capacity. Thus, an innovative functional product with an increase in the quantity of CaLb of $61.4 \%$ with respect to the only such product currently on the market has been developed and could potentially be improved by adding a specific beneficial probiotic $\mathrm{CaLb}$ consumer in order to produce a new symbiotic product.

\section{Acknowledgements}

This work was financially supported by the Principality of Asturias, by the project FC15-GRUPIN14-140. The authors thank Ricardo Alonso Herrero, from Caprinos del Nalón, for providing the milk necessary to manufacture the cottage cheeses.

\section{References}

[1] M. Ashwell, Concepts of functional foods, 2002. doi:10.1108/nfs.2004.34.1.47.3.

[2] H. Ohama, H. Ikeda, H. Moriyama, Health Foods and Foods with Health Claims 
in Japan, Nutraceutical Funct. Food Regul. United States Around World Second Ed. 221 (2014) 265-299. doi:10.1016/B978-0-12-405870-5.00016-5.

[3] S. Alonso, M. Rendueles, M. Díaz, Bio-production of lactobionic acid: Current status, applications and future prospects, Biotechnol. Adv. 31 (2013) 1275-1291. doi:10.1016/j.biotechadv.2013.04.010.

[4] G. Schaafsma, Lactose and lactose derivatives as bioactive ingredients in human nutrition, Int. Dairy J. 18 (2008) 458-465. doi:10.1016/j.idairyj.2007.11.013.

[5] S.S. Suguri T., Yanagidaria S., Kobayashi T., Bifid bacteria multiplicationstimulating agent, 1995. doi:JP07277990A.

[6] FDA. Code of Federal Regulations, Title 21, 21 CFR 172.720. US Food and Drug Administration, 2017. https://www.accessdata.fda.gov/scripts/cdrh/cfdocs/cfcfr/CFRSearch.cfm?fr=172 .720 .

[7] M.I. Dias, I.C.F.R. Ferreira, M.F. Barreiro, Microencapsulation of bioactives for food applications, Food Funct. 6 (2015) 1035-1052. doi:10.1039/c4fo01175a.

[8] L.K. Sarao, M. Arora, Probiotics, prebiotics, and microencapsulation: A review, $\begin{array}{lllllll}\text { Crit. } & \text { Rev. Food }\end{array}$ doi:10.1080/10408398.2014.887055.

[9] T. Morita, Y. Horikiri, T. Suzuki, H. Yoshino, Preparation of gelatin microparticles by co-lyophilization with poly(ethylene glycol): Characterization and application to entrapment into biodegradable microspheres, Int. J. Pharm. 219 (2001) 127137. doi:10.1016/S0378-5173(01)00642-1. 
[10] C.R.L. Francisco, S.A. Heleno, I.P.M. Fernandes, J.C.M. Barreira, R.C. Calhelha, L. Barros, O.H. Gonçalves, I.C.F.R. Ferreira, M.F. Barreiro, Functionalization of yogurts with Agaricus bisporus extracts encapsulated in spray-dried maltodextrin crosslinked with citric acid, Food Chem. 245 (2018) 845-853. doi:10.1016/j.foodchem.2017.11.098.

[11] C. Caleja, A. Ribeiro, L. Barros, J.C.M. Barreira, A.L. Antonio, M.B.P.P. Oliveira, M.F. Barreiro, I.C.F.R. Ferreira, Cottage cheeses functionalized with fennel and chamomile extracts: Comparative performance between free and microencapsulated forms, Food Chem. $199 \quad$ (2016) 720-726. doi:10.1016/j.foodchem.2015.12.085.

[12] P.N. Ezhilarasi, D. Indrani, B.S. Jena, C. Anandharamakrishnan, Microencapsulation of Garcinia fruit extract by spray drying and its effect on bread quality, J. Sci. Food Agric. 94 (2014) 1116-1123. doi:10.1002/jsfa.6378.

[13] B. Cilek, A. Luca, V. Hasirci, S. Sahin, G. Sumnu, Microencapsulation of phenolic compounds extracted from sour cherry pomace: Effect of formulation, ultrasonication time and core to coating ratio, Eur. Food Res. Technol. 235 (2012) 587-596. doi:10.1007/s00217-012-1786-8.

[14] J. Burgain, C. Gaiani, C. Cailliez-Grimal, C. Jeandel, J. Scher, Encapsulation of Lactobacillus rhamnosus GG in microparticles: Influence of casein to whey protein ratio on bacterial survival during digestion, Innov. Food Sci. Emerg. Technol. 19 (2013) 233-242. doi:10.1016/j.ifset.2013.04.012.

[15] M. Premi, H.K. Sharma, Effect of different combinations of maltodextrin, gum arabic and whey protein concentrate on the encapsulation behavior and oxidative stability of spray dried drumstick (Moringa oleifera) oil, Int. J. Biol. Macromol. 
105 (2017) 1232-1240. doi:10.1016/j.ijbiomac.2017.07.160.

[16] Y. Jo, D.M. Benoist, A. Ameerally, M.A. Drake, Sensory and chemical properties of Gouda cheese, J. Dairy Sci. (2017) 1-23. doi:10.3168/jds.2017-13637.

[17] M. Minekus, M. Alminger, P. Alvito, S. Ballance, T. Bohn, C. Bourlieu, F. Carrière, R. Boutrou, M. Corredig, D. Dupont, C. Dufour, L. Egger, M. Golding, S. Karakaya, B. Kirkhus, S. Le Feunteun, U. Lesmes, A. MacIerzanka, A. MacKie, S. Marze, D.J. McClements, O. Ménard, I. Recio, C.N. Santos, R.P. Singh, G.E. Vegarud, M.S.J. Wickham, W. Weitschies, A. Brodkorb, A standardised static in vitro digestion method suitable for food-an international consensus, Food Funct. 5 (2014) 1113-1124. doi:10.1039/c3fo60702j.

[18] S.W. Fatima, S.K. Khare, Current insight and futuristic vistas of microbial transglutaminase in nutraceutical industry, Microbiol. Res. 215 (2018) 7-14. doi:10.1016/j.micres.2018.06.001.

[19] L. Mihalcea, M. Turturică, V. Barbu, E. Ioniţă, L. Pătraşcu, M. Cotârleţ, L. Dumitraşcu, I. Aprodu, G. Râpeanu, N. Stănciuc, Transglutaminase mediated microencapsulation of sea buckthorn supercritical $\mathrm{CO}_{2}$ extract in whey protein isolate and valorization in highly value added food products, Food Chem. 262 (2018) 30-38. doi:10.1016/j.foodchem.2018.04.067.

[20] F. Tello, A.S. Prata, R.A.F. Rodrigues, A. Sartoratto, C.R.F. Grosso, Improving the performance of transglutaminase-crosslinked microparticles for enteric delivery, Food Res. Int. 88 (2016) 153-158. doi:10.1016/j.foodres.2016.02.020.

[21] N.V.N. Jyothi, P.M. Prasanna, S.N. Sakarkar, K.S. Prabha, P.S. Ramaiah, G.Y. Srawan, Microencapsulation techniques, factors influencing encapsulation 
$\begin{array}{lllll}\text { efficiency, } & \text { J. } & \text { Microencapsul. } & 27 & \text { (2010) }\end{array}$ doi:10.3109/02652040903131301.

[22] V. Kaushik, Y.H. Roos, Limonene encapsulation in freeze-drying of gum Arabicsucrose-gelatin systems, LWT - Food Sci. Technol. 40 (2007) 1381-1391. doi:10.1016/j.lwt.2006.10.008.

[23] T.K. Głąb, J. Boratyński, Potential of Casein as a Carrier for Biologically Active Agents, Top. Curr. Chem. 375 (2017). doi:10.1007/s41061-017-0158-Z.

[24] M.A. Soleiman Abbasi, Somayeh Rahimi, Influence of microwavemicroencapsulated citric acid on some sensory properties of chewing gum, J. Microencapsul. 26 (2009) 90-96.

[25] C.G. da Rosa, C.D. Borges, R.C. Zambiazi, M.R. Nunes, E.V. Benvenutti, S.R. da Luz, R.F. D’Avila, J.K. Rutz, Microencapsulation of gallic acid in chitosan, $\beta$ cyclodextrin and xanthan, Ind. Crops Prod. 46 (2013) 138-146. doi:10.1016/j.indcrop.2012.12.053.

[26] V. Chandrasekar, J.N. Coupland, R.C. Anantheswaran, Characterization of nisin containing chitosan-alginate microparticles, Food Hydrocoll. 69 (2017) 301-307. doi:10.1016/j.foodhyd.2017.02.011

[27] I. Stratulat, M. Britten, S. Salmieri, P. Fustier, D. St-Gelais, C.P. Champagne, M. Lacroix, Enrichment of cheese with bioactive lipophilic compounds, J. Funct. Foods. 6 (2014) 48-59. doi:10.1016/j.jff.2013.11.023.

[28] C. García, L. Bautista, M. Rendueles, M. Díaz, A new synbiotic dairy food containing lactobionic acid and Lactobacillus casei, Int. J. Dairy Technol. 70 (2018) 1-10. doi:10.1111/1471-0307.12558. 
[29] T. Kiryu, T. Kiso, H. Nakano, K. Ooe, T. Kimura, H. Murakami, Involvement of Acetobacter orientalis in the production of lactobionic acid in Caucasian yogurt (“Caspian Sea yogurt”) in Japan, J. Dairy Sci. 92 (2009) 25-34. doi:10.3168/jds.2008-1081.

[30] C. Wilkinson, G.B. Dijksterhuis, M. Minekus, From food structure to texture, Trends Food Sci. Technol. 11 (2001) 442-450. doi:10.1016/S09242244(01)00033-4.

[31] E.A. Foegeding, M.A. Drake, Invited Review: Sensory and Mechanical Properties of Cheese Texture, J. Dairy Sci. 90 (2007) 1611-1624. doi:10.3168/jds.2006-703.

[32] D.W. Everett, M.A.E. Auty, Cheese structure and current methods of analysis, Int. Dairy J. 18 (2008) 759-773. doi:10.1016/j.idairyj.2008.03.012.

[33] Z. Pang, H. Deeth, P. Sopade, R. Sharma, N. Bansal, Rheology, texture and microstructure of gelatin gels with and without milk proteins, Food Hydrocoll. 35 (2014) 483-493. doi:10.1016/j.foodhyd.2013.07.007.

[34] M. Jack, Cheese Analogues, 4 (2011) 814-821.

[35] G. Xing, X. Rui, M. Jiang, Y. Xiao, Y. Guan, D. Wang, M. Dong, In vitro gastrointestinal digestion study of a novel bio-tofu with special emphasis on the impact of microbial transglutaminase, PeerJ. 4 (2016) e2754. doi:10.7717/peerj.2754. 


\section{Figures Caption}

Figure 1. SEM images of (A) NaCas, (B) NaCas-TGase, (C) Gel, (D) Gel-TGase, (E) GA 4\%, (F) GA 8\%, (G) MD 12\%, (H) MD 16\%, (I) GA:MD 75:25, (J) GA:MD 50:50 and (K) GA:MD 25:75 microparticles; scale bars correspond to $100 \mu \mathrm{m}$.

Figure 2. Experimental and simulated evolution of CaLb percentage in cottage cheesemicroparticles mixes with time along 12 days. Points indicate experimental results, $(\diamond)$

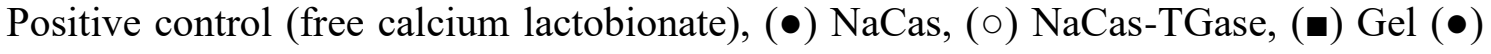
GA 8\%, (ロ) MD 16\%. Lines show simulated evolution using the proposed model.

Figure 3. Flows/reactions and concentration profiles assumed in the experimental system for modelling.

Figure 4. Textural analysis of functionalised cottage cheeses; (A) firmness analysis and (B) stickiness analysis. Both are expressed in force units (g).

Figure 5. SEM images of cheese (A) without microparticles in its structure, (B) with free calcium lactobionate, (C) with CasNa and (D) with NaCas-TGase microparticles; scale bars correspond to $50 \mu \mathrm{m}$. 